\title{
BMJ Open Longitudinal pre-exposure prophylaxis (PrEP) acceptability, initiation and adherence among criminal justice- involved adults in the USA: the Southern PrEP Cohort Study (SPECS) protocol
}

Katherine LeMasters (D) , ${ }^{1,2}$ Carrie Oser, ${ }^{3}$ Mariah Cowell, ${ }^{2,4}$ Katie Mollan, ${ }^{1,5}$ Kathryn Nowotny, ${ }^{6}$ Lauren Brinkley-Rubinstein ${ }^{2}$

To cite: LeMasters $\mathrm{K}$, Oser $\mathrm{C}$, Cowell $\mathrm{M}$, et al. Longitudinal pre-exposure prophylaxis (PrEP) acceptability, initiation and adherence among criminal justice-involved adults in the USA: the Southern PrEP Cohort Study (SPECS) protocol. BMJ Open 2021;11:e047340. doi:10.1136/ bmjopen-2020-047340

- Prepublication history for this paper is available online. To view these files, please visit the journal online (http://dx.doi org/10.1136/bmjopen-2020047340).

Received 25 November 2020 Accepted 07 July 2021

Check for updates

(c) Author(s) (or their employer(s)) 2021. Re-use permitted under CC BY-NC. No commercial re-use. See rights and permissions. Published by BMJ.

For numbered affiliations see end of article.

Correspondence to Katherine LeMasters; katherine.lemasters@unc.edu

\section{ABSTRACT}

Introduction HIV prevalence among criminal justice (CJ)-involved adults is five times higher than the general population. Following incarceration, CJ-involved individuals experience multilevel barriers to HIV prevention. Preexposure prophylaxis (PrEP) is a widely available, daily medication efficacious in preventing HIV. Little is known about PrEP knowledge, acceptability, initiation and sustained use among CJ-involved persons or about how these outcomes vary by multilevel factors. The Southern Pre-Exposure Prophylaxis Study (SPECS) will investigate barriers and facilitators for PrEP initiation and sustained use among CJ-involved adults, building a foundation for PrEP interventions for this underserved population.

Methods and analysis SPECS uses a mixed-methods sequential design, including a multisite, prospective cohort study in three southern states-North Carolina, Florida and Kentucky — and subsequent qualitative interviews. HIV-negative adults clinically indicated for PrEP with CJinvolvement in the past year ( $n=660 ; 220$ per site) —will be recruited for four quantitative interviews separated by 6 months, with 18 months of follow-up. Interviews will measure $\mathrm{CJ}$ involvement, substance use, sexual behaviours, PrEP acceptability and use, healthcare access and utilisation, support systems and psychological and emotional well-being. We will estimate probabilities of PrEP acceptability and use in a CJ-involved population using descriptive and multivariable analyses. After the follow-up, a subsample that never initiated PrEP, initiated but did not sustain PrEP or sustained PrEP will be asked to participate in a qualitative interview to contextualise their experiences and decisions around PrEP. An inductive approach will guide qualitative analyses.

Ethics and dissemination PrEP initiation and sustained use rates are unknown among CJ-involved adults. This research will identify individual, social and structural factors that predict PrEP initiation and use. Data generated from the study have the potential to guide research and the development and tailoring of PrEP interventions to CJ-involved populations and provide context to HIV-related outcomes for those with CJ experiences.

\section{Strengths and limitations of this study}

- This is the first pre-exposure prophylaxis (PrEP)related multisite, observational cohort among a criminal justice (CJ)-involved population, which is important as rates of PrEP initiation and sustained use remain unknown among those with recent $\mathrm{CJ}$ experience.

- This study will collect longitudinal data relevant to the individual, social and structural level for CJinvolved populations and will include HIV risk profiles typically excluded from studies on CJ-involved populations, including men who have sex with men.

-We are focused on populations in the southern USA, which sit at the intersection of a number of HIV risk factors and have received less attention than other regions of the USA.

- We are relying primarily on self-reported PrEP initiation and sustained use, which are susceptible to recall bias.

- It is possible that few participants will initiate or sustain PrEP.

\section{INTRODUCTION}

In the USA, 1 in every 38 adults is actively involved in the criminal justice (CJ) system. ${ }^{1}$ Of the 6.6 million CJ-involved adults, over two-thirds or 4.5 million, are under a form of community-based supervision such as probation or parole. ${ }^{1}$ While incarceration rates for white Americans is increasing, the CJ system continues to disproportionally affect persons of colour (POC). In particular, black and Latinx adults are significantly overrepresented in incarcerated populations. Although they represent $12 \%$ and $16 \%$ of the adult population in the USA, respectively, black and Latinx Americans make up 33\% 
and $23 \%$ of the adult prison population. ${ }^{23}$ Racial disparities in incarceration rates are stark among young black men aged $18-19$, who are 12 times more likely to experience incarceration than their white peers. ${ }^{3}$

Vulnerable populations, such as those experiencing poverty, homelessness, severe and persistent mental illness or substance use disorders, are frequently trapped by the revolving doors of the $\mathrm{CJ}$ system and experience multiple periods of incarceration in their lifetime. ${ }^{4-6}$ Increasingly, as a consequence of the opioid epidemic, there is a strong relationship between the intensity of opioid use and history of $\mathrm{CJ}$ involvement among people who inject drugs (PWIDs) ${ }^{7}$ Therefore, it is unsurprising that the American South, which sees a confluence of racial discrimination, poverty and disenfranchisement, has incarceration rates among the highest in the nation. ${ }^{8-10}$ Notably, we also see the disparities in mass incarceration reflected in risks of HIV transmission. There is a high concentration of those with-or at risk of-HIV infection concentrated in the US correctional system.

HIV prevalence among CJ-involved adults is five times that of the general population, and an estimated one in seven individuals infected with HIV pass through the CJ system each year. ${ }^{1112}$ While incarcerated, CJ-involved individuals are at risk of coming in contact with HIV through unsterile needle practices, tattooing and condomless sex. Following periods of incarceration, individuals may also rapidly re-engage with risky behaviour that increases the risk of transmission, including injection drug use and condomless sex, making periods following incarceration high risk for community-based HIV transmission. ${ }^{13-15}$ In the American South, where, like incarceration, HIV prevalence is among the highest in the USA, black Americans account for $54 \%$ of new HIV diagnoses. ${ }^{16}$ It is clear that the collateral effects of incarceration on HIV risk among POC, including creating and perpetuating imbalanced gender ratios in communities due to the disproportionate incarceration of Black men and thus facilitating partnership concurrency, and disrupting access to healthcare, contribute to disproportionally high rates of HIV among this population. ${ }^{11} 1417-19$ As identified in a 2016 report from the Centers for Disease Control and Prevention (CDC), the South's high rates of opioid use, HIV stigma, poverty levels, and poor healthcare infrastructure have created a perfect storm for an HIV outbreak. ${ }^{16}$ In particular, HIV stigma in the South around sexual orientation, substance use, poverty and sex work limits people disclosing their HIV status, adhering to antiretroviral medications and seeking healthcare. ${ }^{920}$ Despite this, HIV prevention efforts thus far, including interventions to link people to HIV care postrelease and risk reduction interventions, have led to little meaningful change in reducing rates of infection in CJ-involved persons in the South.

The failure of traditional approaches (eg, promoting HIV testing and the use of condoms) to address expanding risk of HIV infections among people most at risk has led to new HIV prevention strategies that combine behavioural and biomedical approaches. In addition to early diagnosis and sustained treatment of HIV infection, daily preventative treatment-pre-exposure prophylaxis (PrEP) emtricitabine/tenofovir (FTC/TDF) - has been credited with recent reductions in rates of new HIV diagnoses in the USA. ${ }^{21}$ Efficacy of PrEP has been established in randomised control trials and open label studies and is approved by the FDA for HIV prevention. ${ }^{22}{ }^{23} \mathrm{PrEP}$ has been recommended by the CDC for any at-risk population, including men who have sex with men (MSM), PWIDs and heterosexuals who engage in condomless sex with at risk populations. The PrEP care cascade includes identifying those at highest risk for HIV, increasing HIV and PrEP awareness among those individuals, initiating PrEP, and retaining individuals in PrEP care. ${ }^{24}$

Despite its efficacy, PrEP uptake has been slow. It is estimated that 1.1 million Americans are candidates for PrEP-those engaging in high risk sex or unsafe injection drug use-yet, current reports indicate that as few as 7\% of PrEP candidates have been prescribed PrEP. ${ }^{25}{ }^{26}$ Little is known about PrEP use among those on post-release supervision/parole and probation, and challenges to PrEP use among these populations is abundant. To be most efficacious, oral PrEP must be taken daily. Additionally, as a prescribed medication, PrEP must be offered to candidates in a clinical setting, followed by regular monitoring for adverse effects, sustained use and sexually transmitted infections (STIs) including HIV. Procurement of PrEP also requires navigating systems to cover the cost of the medication and attendant testing. A major additional challenge is an under appreciation of HIV acquisition risk among those who the WHO consider to be at substantive risk. Substantive risk is defined as individuals belonging to a group that has a disproportionate burden of HIV, which includes CJ populations. ${ }^{27}$ Therefore, despite its promise, PrEP is underutilised and understudied in one of the most at-risk populations in the US-individuals with recent $\mathrm{CJ}$ involvement.

\section{Objectives}

This paper describes The Southern Pre-Exposure Prophylaxis Study (SPECS), which uses a mixed-methods sequential design. SPEC includes a prospective observational cohort study, followed by qualitative interviews which are designed to respond to and close the knowledge gap regarding PrEP and those involved in the CJ system. SPECS is a multisite, 5-year study that will take place in three, diverse southern settings: North Carolina, Kentucky, and Florida. The specific aims of this study are to: (1) characterise PrEP knowledge and acceptability among those placed on postrelease supervision/ parole or probation within the last 12 months, (2) identify the multilevel factors that predict PrEP initiation and sustained use among those placed on post-release supervision/parole or probation within the last 12 months and (3) qualitatively assess the multilevel factors that affect PrEP acceptability, initiation and sustained use to inform future intervention development. 


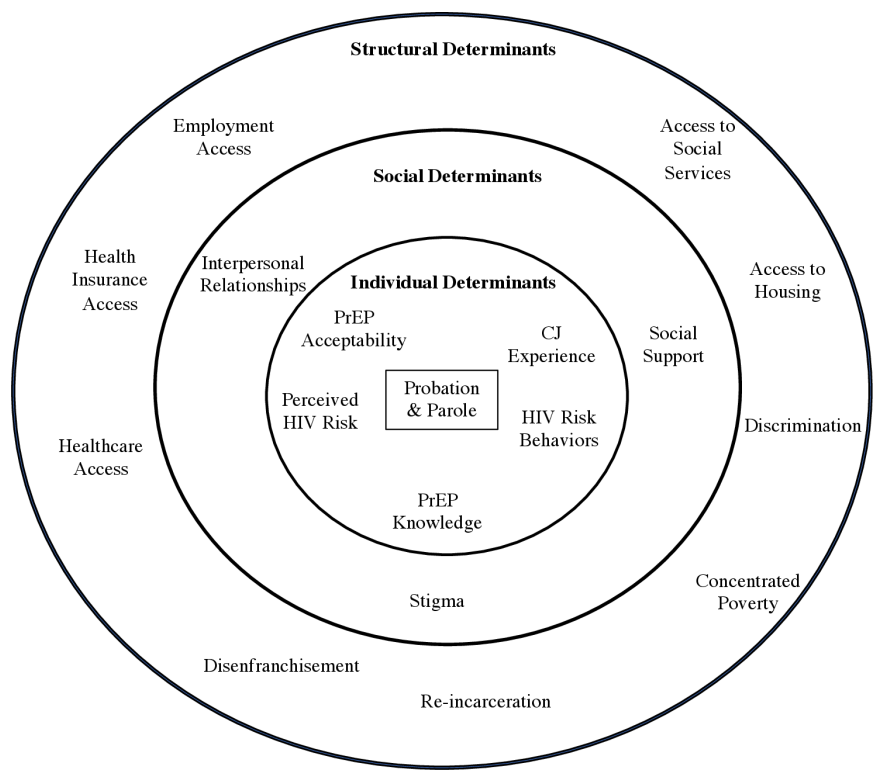

Figure 1 The social ecological model of PreP use. CJ, criminal justice; PrEP, pre-exposure prophylaxis.

\section{Broad goals for the study}

The significance of the study is punctuated by the confluence of the continuing dual epidemics of incarceration and HIV. Very few studies have examined knowledge, acceptability, initiation and sustained use of PrEP among individuals with CJ involvement. Importantly, the proposed investigations will identify the multilevel barriers and facilitators that predict PrEP initiation and sustained use among parolees and probationers, building the foundation for next-step PrEP interventions for this largely underserved population.

\section{Conceptual framework}

Both the socioecological model and the National Institute of Minority Health and Health Disparpities research framework inform SPECS, ${ }^{28}{ }^{29}$ centralising the importance of health disparities that span multilevel domains of influence. Specifically, these domains extend beyond the individual to consider the importance of social and structural factors and their responsibility for reducing health inequalities. $^{2829}$ These frameworks will be used in combination to observe and study the longitudinal patterns of PrEP initiation and sustained use among people on probation and parole, who are disproproportionately POC and experience health disparities (figure 1).

\section{METHODS AND ANALYSES \\ Study design}

This study is a prospective longitudinal cohort of individuals with CJ involvement and a clinical indication for PrEP in North Carolina, Florida and Kentucky.

\section{Participants}

Participants will be HIV negative adults over the age of 18 who were placed on postrelease supervision/parole or probation within the last 12 months. Eligible participants will be at substantial risk for HIV infection based on CDC/ WHO criteria for PrEP eligibility: PWIDs, persons with recent bacterial STI diagnosis, and/or individuals who are at a higher risk for being exposed to HIV through sex, including MSM and those with infrequent condom use.

\section{Recruitment}

Participants will be recruited at three study sites with the following enrolment goals: NC $(n=220), K Y(n=220)$ and FL $(n=220)$. Recruitment will occur at local probation and parole offices in Durham and Raleigh, NC, Miami, FL and Lexington, KY. Study team members will approach individuals in the waiting room at each local probation and parole office, and screen in a private on-site room. Participants will provide written informed consent. Participants may understandably feel uncomfortable answering questions about sensitive subjects like sexual activity and substance use while at a probation and parole office. They will be provided with the option of completing prescreen surveys over the phone or at an off-site venue, such as the university or study community office.

\section{Quantitative data collection}

Standard study assessments will be conducted at baseline, 6 months, 12 months and 18 months, using Research Electronic Data Capture programmed surveys. At all timepoints, participants will answer questions related to the three domains in the social ecological model of PrEP use, ${ }^{25} 26$ including items on individual level factors (ie, sociodemographic information; CJ involvement; substance use; sexual risk behaviour; PrEP acceptability, initiation, and sustained use; medical comorbidities; healthcare utilisation), social level factors (eg, stigma and discrimination; social support ${ }^{30}$ ) and structural level factors (healthcare access; housing access; employment; public transportation). Participants will also be tested for HIV at baseline and at the end of the study at 18-month follow-up with an OraQUICK ADVANCE Rapid HIV-1/2 test. When HIV tests are administered at baseline and 18 months, participants will also receive pretest, posttest and PrEP counselling. All participants that receive a positive HIV test will receive support to access HIV care; however, those that receive a positive HIV test at baseline are ineligible for study enrolment. At baseline, participants will complete a locator form for which information will be updated during phone calls at 3 months, 9 months and 15 months. This form contains contact information for the participant (eg, phone numbers, address, social media handles) as well as for key family or friends. Participants will receive US $\$ 40$ per interview and US $\$ 10$ per phone call. Participants completing all interviews and phone calls will receive US $\$ 190$.

\section{Retention and attrition}

We recognise that CJ-involved populations are difficult to retain in longitudinal studies (ie, due to recidivism, substance use, unstable housing), but our research team has been successful in limiting study attrition among 
CJ-involved populations. ${ }^{32} 33$ Therefore, we postulate that we will be able to retain $80 \%$ of our study population for 18-month follow-up.

This study will employ multiple processes to support participant retention over 18 months of data collection. These strategies operate at both the individual and organisational level. At the individual level, we will do same-day screening, enrolment and assessment when possible. Every 3 months, we will check-in with participants by phone, email, and field contact and will subsequently update locater information forms and send letters and postcards. Throughout the study, we will send personalised notes to participants (ie, birthdays, holidays), schedule appointments outside of their work hours and in the community that is most convenient for them, provide monetary incentives, establish a study Facebook page, and conduct active case-finding for persons lost to follow-up (ie, internet searches, home visits). At the organisational level, we will develop a study logo and branding, conduct weekly automated prison and jail database searches, and have regular team conference calls. At year one, we will have a retention training workshop and conduct study and site training.

Each site will tailor or add additional methods based on experience and resources and sites will continually communicate with each other to ensure adherence to all retention methods. Additionally, through engaging in automated prison and jail database searches, we will track participants in real time as they experience incident reincarceration. Individuals that become reincarcerated will remain in the study if they are able to attend scheduled study visits after incarceration.

\section{Study exposures and outcomes}

Our outcomes for aim 1 are PrEP knowledge and acceptability. PrEP knowledge will be assessed by asking participants if they have prior knowledge of PrEP and PrEP acceptability will be assessed using the Willingness to Use PrEP scale. ${ }^{34} 35$ Our outcomes for aim 2 are PrEP initiation and sustained use. PrEP initiation is a dichotomous measure defined as receiving/filling a prescription for PrEP as reported at the first follow-up assessment (at 6 months). Sustained use is defined as self-report daily adherence assessed by the number of PrEP doses taken in the last 30 days. This measure of adherence will be collected at 6,12 and 18 months follow-ups. Self-reported adherence will be supplemented by clinical chart abstraction. Secondary outcomes for aim 2 are HIV seroconversion ( 20 seroconversions are anticipated) as well as dichotomous and count measures of non-PrEP-related health service utilisation (eg, emergency department, other preventative care) and behaviours associated with high HIV risk. A combination of individual (eg, healthcare utilisation), social (eg, social support) and structural factors (eg, re-incarceration) serve as exposure variables of interest.

The study is using a sequential design in which aim 3 builds on aims 1 and 2 to help elucidate patterns of PrEP use and multilevel factors that facilitate or act as barriers to PrEP knowledge, initiation, and sustained use. Specifically, aim 3 includes qualitative interviews which are designed to illicit a rich descriptive narrative regarding the PrEP continuum of care. Example questions include 'Describe how concerned you are that other people know you are on (or might be considering) PrEP?" or "If we were going to create an intervention that would help support you in getting your PrEP prescriptions, getting to the clinic, and taking PrEP every day, what do you think would be helpful?'

\section{Sample size calculations}

Retention of participants is assumed to be $80 \%$ at each follow-up through 18 months (ie, 528 evaluable at each follow-up). A primary objective of Aims 1 and 2 is to estimate the probabilities of PrEP knowledge, acceptability, initiation and sustained use, overall and at each site. The anticipated proportions having these outcomes are unknown. Table 1 provides precision calculations for observed proportions ranging from $50 \%$ to $90 \%$. Power to detect associations depends on the true probabilities of PrEP knowledge, acceptability, initiation and sustained use is shown in table 2.

For example, analysing the full cohort and a covariate with $50 \%$ prevalence, there will be sufficient power $(>80 \%)$ to detect an OR of 2.0 or larger at a single time point for a reference group proportion $(\mathrm{P})$ with the outcome event (eg, PrEP initiation) ranging from $20 \%$ to $80 \%$ (table 2 presents P: $50 \%-80 \%$ ). For site-specific

Table 1 Precision calculations for proportions with PreP knowledge, acceptability and initiation

\begin{tabular}{|c|c|c|c|}
\hline \multicolumn{2}{|c|}{ Full cohort (528 evaluable) } & \multicolumn{2}{|c|}{ Site-specific (176 evaluable) } \\
\hline Proportion $(95 \% \mathrm{Cl})$ & Cl half-width & Proportion $(95 \% \mathrm{Cl})$ & $\mathrm{Cl}$ half-width \\
\hline 0.5 (0.46 to 0.54$)$ & 0.043 & $0.5(0.42$ to 0.58$)$ & 0.076 \\
\hline 0.7 (0.66 to 0.74$)$ & 0.040 & 0.7 (0.63 to 0.77$)$ & 0.070 \\
\hline 0.8 (0.76 to 0.83$)$ & 0.035 & 0.8 (0.73 to 0.86$)$ & 0.061 \\
\hline
\end{tabular}

Exact binomial $95 \%$ Cls are shown.

PrEP, pre-exposure prophylaxis. 
Table 2 Power to detect association between an independent variable with $50 \%$ prevalence and PrEP outcomes ( $\mathrm{N}=528$ evaluable)

\begin{tabular}{lllll}
\hline OR & $\mathbf{P}=\mathbf{0 . 5}$ & $\mathbf{P}=\mathbf{0 . 6}$ & $\mathbf{P}=\mathbf{0 . 7}$ & $\mathbf{P}=\mathbf{0 . 8}$ \\
\hline 1.75 & 88.7 & 85.6 & 78.6 & 65.0 \\
2.00 & 97.3 & 95.8 & 91.7 & 80.9 \\
2.25 & 99.5 & 98.9 & 97 & 90.0 \\
\hline
\end{tabular}

$\mathrm{p}=$ proportion with the outcome (eg, PrEP initiation) in the reference group.

PrEP, pre-exposure prophylaxis.

analyses, an OR of 3.5 or larger at a given time point will be detectible with $>85 \%$ power for $\mathrm{P}$ ranging from $10 \%$ to $70 \%$. Longitudinal analyses incorporating measurements from baseline, 6, 12, and 18 months will have higher power that will be impacted by within-individual correlations over time. Analyses of sustained PrEP use will be among the subsample who initiate PrEP and site-specific analyses of sustained PrEP will be descriptive.

\section{Qualitative data collection}

SPECS cohort participants will be categorised into the following three groups for each site: never initiated PrEP, initiated but did not sustain and initiated and sustained. Research randomiser (www.randomizer.org) will be used to randomly select eight or nine individuals from each of the three groups to participate in the qualitative interview. Enrollment will continue until data analysis reflects saturation, with 25 interviews anticipated at each study site and approximately an equal number of interviews of parolees who (1) never initiated PrEP, (2) initiated PrEP but did not sustain use and (3) initiated and sustained use of PrEP. Participants will receive US $\$ 40$ for completing the interview. Interviews will take place in year 4 and 5 so the qualitative sample will include those who had long term, sustained use of PrEP. In-depth qualitative interviews will last $60-90 \mathrm{~min}$ and will take place face-to-face, in a private room at a community-based venue convenient for the participant. All interviews will be digitally recorded and professionally transcribed. Team members who have training and expertise in qualitative research will conduct the interviews. Content of interviews will be guided by the conceptual framework, our research objectives, and findings from the quantitative data collected via aims 1 and 2. However, we expect to explore the domains below:

1. Risk compensation. Example questions will include: (1) Has your HIV risk behaviour changed over time, recently, please explain? For instance, is there a period in the past when you were engaging in risky behaviour and then a time when you thought you definitely were not? Can you tell me about those times? (2) Do you think use of PrEP affected your risk engagement, why/ why not?

2. Knowledge, acceptability and experience related to PrEP initiation/sustained use. Example questions will include: (1) Can you tell me a little bit about your ex- perience with PrEP (prompts for follow-up will include questions relevant to the PrEP clinic and provider, their experience accessing PrEP clinic visits and availability of PrEP in their community, etc); (2) When you think about making the decision to stay on PrEP or not take PrEP, what comes to mind as something that helped you make that decision?

3. The multilevel barriers/facilitators of PrEP initiation/ sustained use and suggestions for future PrEP intervention components. Example questions will include: (1) Earlier I asked if anything you had been dealing with might be affecting your HIV risk behaviour, similarly, do you think any of the things you've been dealing with might have affected your PrEP use, why/why not?; (2) Were you ever concerned what other people would think if they knew you were on (or might be considering) PrEP? (3) If we were going to create an intervention that would help support you in getting your PrEP prescriptions, getting to the clinic, and taking PrEP every day, what do you think would be helpful?

4. Interest in new PrEP modalities. Example questions will include: (1) There are many different emerging models for how to get PrEP. Would you be interested in getting PrEP from a shot that you only had to get every few months, why/why not? (2) Would you be interested in getting your PrEP prescription in the mail, why/ why not? (3) Would you be interested in completing a self-HIV test at home every 3 months and mailing it to the clinic instead of having to have an in person visit, why/why not? (4) Which of these options would you most prefer?

\section{Quantitative analyses}

Data analysis will be guided by our conceptual framework, which postulates that PrEP knowledge, acceptability, initiation and sustained use are impacted by multilevel factors at the individual, social and structural levels. The multilevel nature of these factors will be considered when constructing analytic models. Quantitative analyses will be conducted using the full cohort of 660 participants and also evaluated within each site ( $\mathrm{n}=220$ per site) to elucidate state-specific outcomes. Both aims 1 and 2 will use a descriptive, bivariate and multivariable approach for analyses. Aim 1 multivariable analyses will use (1) generalised linear models (GLM) to estimate associations between independent variables (ie, exposures) and the outcomes of (A) PrEP knowledge and (b) average/ high PrEP acceptability. We will estimate model-based predicted probabilities of PrEP acceptability based on key independent variables (eg, sex, race/ethnicity, sexual identity, CJ history, MSM, IDU) to elucidate key subpopulations where future interventions are needed to improve PrEP acceptability. Aim 2 will conduct multivariable analyses using GLMs to analyse longitudinal data for sustained PrEP use, and will examine results at each follow-up time point. Generalised estimating equations with robust SE estimates ${ }^{36}$ will be used to estimate parameters of a GLM; a priori we will assume an exchangeable working 
correlation structure. Covariate associations with PrEP initiation, time-to-initiation and number of PrEP clinic appointments kept will also be analysed. We will estimate associations between individual, social, and structural factors and time to initiation of PrEP, with proper handling of right-censored observations for those who do not initiate PrEP. ${ }^{38}$ While our primary outcome of PrEP initiation will be measured at 6-month follow-up, PrEP initiation can be examined at all follow-up time points. Additional secondary outcomes of interest include: HIV seroconversion ( $\sim 20$ seroconversions are anticipated), non-PrEP-related health service utilisation, reincarceration and substance use. Missing data will be an important consideration, and will be handled in multivariable analyses using a missing at random assumption, when deemed tenable, via application of multiple imputation or inverse probability of missingness weights. ${ }^{40-42}$ If data are suspected to be missing not at random, this will be described as a limitation.

\section{Qualitative analyses}

Aim 3 will qualitatively assess the multi-level factors that affect PrEP acceptability, initiation and sustained use to inform future intervention development. A general inductive approach guided by the conceptual framework and the research objectives will be used. ${ }^{43}$ A preliminary codebook will be developed after the first three transcripts from each category (eg, those who never started PrEP, those who initiated but did not sustain PrEP use, and those who sustained PrEP use) are complete. The coding scheme will be tested and two coders will code the text segments, compare coding and discuss and resolve areas of discrepancy, and then code another 20 text segments and percentage agreement will be calculated. Open coding will be done first followed by broad thematic analysis. Transcripts will be coded with axial codes, or more interpretive codes, that will be used in order to identify core concepts. In addition, memos and theory notes will be generated throughout the analysis.

\section{Timeline}

During the Fall of 2019, we will begin cohort recruitment and follow-up, which will continue through the Summer of 2022. Aim 1 analysis will take place in 2021-2022 while aim 2 analysis will take place in 2021-2023. Aim 3 protocol development and recruitment will take place in the second half of 2021 while aim 3 data collection and analysis will take place in 2022-2023. Dissemination of findings will take place in 2021-2023.

\section{Patient and public involvement \\ No patients involved.}

\section{The coronavirus pandemic}

Due to the coronavirus pandemic, we will adjust our recruitment and retention efforts. In March of 2020, in-person recruitment and retention will pause and transition to conduct all retention virtually through phone calls. For retention, we will send personalised mailers to enrolled participants, pay participants for contacting SPECS' staff, and give participants a bonus for completing their retention visits within a week of when they were scheduled. We will also begin a coronavirus survey with an additional cash incentive for enrolled participants. With additional incentives, particpants will go from earning up to US\$190 to earning up to US\$265 for completing SPECS.

Recruitment will be paused beginning March 2020. When feasible, we will resume recruitment using chain referral, asking enrolled participants to refer up to five potentially eligible peers to us (ie, anyone placed on probation, postrelease supervision or parole in the past 12 months). Enrolled participants will receive US $\$ 10$ for each referral, regardless of if the referalls qualify for the study. Qualifying individuals will complete the baseline interview over the phone. Once it is safe to do so, we will return to in-person recruitment and retention.

\section{ETHICS AND DISSEMINATION}

While PrEP is highly effective at preventing HIV, it will be most effective if used by those for whom traditional approaches for HIV prevention have failed. HIV prevalence among CJ-involved populations remains five times that of the general population, yet little remains known about PrEP uptake among CJ-involved individuals and about facilitators and challenges to PrEP uptake and sustained use. ${ }^{12}$ By adopting a conceptual framework that recognises PrEP decisions are made within individual, social and structural levels, SPECS is designed to respond to and close the knowledge gap regarding PrEP among people involved in the CJ system.

The SPECS cohort will establish a platform to support future research related to PrEP outcomes among parolees. We suspect that future research could include: (1) Comparison of PrEP-related outcomes among cohort members who do and do not become re-incarcerated during the study period; (2) Spatial epidemiological analyses using geographic information derived from offender databases and collected from cohort participants and (3) Study of the possible effect of Medicaid expansion in $\mathrm{KY}$ and non-expansion of Medicaid in FL and NC. Similarly, we can compare across states for other outcomes. In terms of future interventions, we anticipate the results of SPECS will highlight specific needs that should be addressed for certain subgroups. For example, our results could indicate that some subgroups might be more likely to initiate PrEP but less likely to engage in sustained use-identifying a key intervention time point in the PrEP care cascade. We could also find that certain groups experience very specific barriers to PrEP use postrelease, identifying the need to provide tailored support. Therefore, SPECS will lay the groundwork for future randomised clinical trials to evaluate the efficacy of multiple PrEP-related interventions that are tailored for CJ-involved individuals. 


\section{Ethical concerns}

A primary ethical concern is that we are collecting highly sensitive, personal information from a population that often experiences stigma and discrimination and is at high risk for recidivism. Specifically, we collect information on activities that are illegal, notably illicit drug use. Recognising this, the collection of identifiable information will be minimised to the greatest extent possible. Extensive efforts will be dedicated to protecting the confidentiality and privacy of study participants. Specifically, all data will be stored in a deidentified format and only accessed by the study team. Participants will be thoroughly briefed on these protections as part of the consent process. The study's protocol and procedures have been reviewed and approved by the institutional review boards at the University of North Carolina at Chapel Hill (18-2466). In addition, participants are protected under a federal Certificate of Confidentiality.

Additionally, the baseline assessment may take place in private rooms in probation and parole offices, or a community venue of the participant's choice (eg, private room at the university or a community-based organisation). The baseline assessment at the probation and parole office is often ideal, as we can recruit from the waiting room and each site has been given a private space to screen and conduct the baseline assessment. However, we acknowledge that it will be important to reassure participants, during the consent procedure that their answers will be confidential, and issues discussed will in no way impact their CJ status, nor ability to obtain or be referred to medical care or social services. In addition, we will not share the purpose of the study with individual probation or parole officers. Instead, this study will be referred to broadly as a 'health study'.

\section{Methodological limits and concerns}

This study has methodological limitations that merit further discussion. First, it is possible that we will not recruit the number of participants needed at each site. If this is the case, we will expand recruitment to additional district offices in each site location. In addition, if needed, we will expand to recruit from communitybased organisations. Similarly, it is possible that we retain fewer than $80 \%$ of participants. If this is the case, we will redirect the duties of one research staff member at each site to focus solely on retention efforts. If retention is below $80 \%$, we will also increase the dollar amount of the follow-up incentives. Second, while we assume a sizeable portion of our cohort will initiate and sustain PrEP, the proportion may be low. As a part of our power calculations, we have estimated a wide range of possible outcome events. Therefore, even if the number of individuals who initiate and sustain PrEP is low, we should have the power to perform our planned analyses. In addition, if we find that few people initiate and sustain PrEP, we will reorient aim 3 to focus on the reasons for non-initiation or unsustained use. Third, while the most robust PrEP adherence measure would be to measure drug concentration in dried blood spots (DBS), this is very expensive and typically not feasible for real-world PrEP observational studies. We are, therefore, assessing adherence using self-reported number of missed doses in the past 30 days. Recall over 30-day periods is commonly used as a measure of adherence, and these measures have demonstrated reliability when compared with objective measures of adherence. In addition, this adherence measure has been found to be highly consistent with drug concentrations found using DBS analysis of PrEP. ${ }^{44}$ Lastly, cohort studies may bias, due to recall and social desirability bias of self-reported measures, in-selection bias due to HIV-related stigma, out-selection bias due to attrition, and data missing not at random.

\section{CONCLUSIONS}

There are many reasons to study the health of CJ-involved populations in the US South, specific to and beyond HIV prevention. The CJ system and HIV continue to disproportionally affect POC and vulnerable populations, and these effects are strongest in the US South. While SPECS aims to contribute to the growing body of literature on PrEP uptake and retention among this population, it also aims to address inequities in research, specifically the paucity of data that can be used to improve the lives and health of CJ-involved populations at risk of HIV. This study's dataset will provide the potential to answer related questions to reduce the risk of HIV and ultimately improve the lives of these individuals.

\section{Author affiliations \\ ${ }^{1}$ Department of Epidemiology, University of North Carolina at Chapel Hill, Chapel Hill, North Carolina, USA \\ ${ }^{2}$ School of Social Medicine, Center for Health Equity Research, University of North Carolina at Chapel Hill, Chapel Hill, North Carolina, USA \\ ${ }^{3}$ Department of Sociology, Center on Drug \& Alcohol Research, Center for Health Equity Transformation, University of Kentucky, Lexington, Kentucky, USA \\ ${ }^{4}$ School of Social Work, University of North Carolina at Chapel Hill, Chapel Hill, North Carolina, USA \\ ${ }^{5}$ Center for AIDS Research, University of North Carolina at Chapel Hill School of Medicine, Chapel Hill, North Carolina, USA \\ ${ }^{6}$ Department of Sociology, University of Miami, Miami, Florida, USA}

Acknowledgements This research would not have been possible without the North Carolina Department of Public Safety, Kentucky Department of Corrections, and Florida Department of Corrections participation; however, the findings and ideas presented are solely those of the authors.

Contributors KL: drafted and revised manuscript; approved final submission. C0: revised manuscript; substantially contributed to the conception and design and analytic approach; approved final submission. MC: revised manuscript; approved final submission. KM: revised manuscript; substantially contributed to the analytic approach; approved final submission. KN: revised manuscript; substantially contributed to the conception and design and analytic approach; approved final submission. LB-R: revised manuscript; substantially contributed to the conception and design and analytic approach; approved final submission.

Funding This work is supported by The NIMHD (1R01MD013573-01) and by The University of North Carolina at Chapel Hill Centre for AIDS Research (CFAR), an NIHfunded programme P30 Al050410.

Competing interests None declared.

Patient and public involvement Patients and/or the public were not involved in the design, or conduct, or reporting, or dissemination plans of this research. 
Patient consent for publication Not required.

Provenance and peer review Not commissioned; externally peer reviewed.

Open access This is an open access article distributed in accordance with the Creative Commons Attribution Non Commercial (CC BY-NC 4.0) license, which permits others to distribute, remix, adapt, build upon this work non-commercially, and license their derivative works on different terms, provided the original work is properly cited, appropriate credit is given, any changes made indicated, and the use is non-commercial. See: http://creativecommons.org/licenses/by-nc/4.0/.

\section{ORCID iD}

Katherine LeMasters http://orcid.org/0000-0002-1754-1730

\section{REFERENCES}

1 Kaeble D, Cowhig M. Correctional populations in the United States, 2016, 2018.

2 Gramlich J. Black imprisonment rate in the U.S. has fallen by a third since 2006. Pew Research Center, 2020.

3 Carson A. Prisoners in 2018 [Internet], 2019. Available: https://www. bjs.gov/index.cfm?ty=pbdetail\&iid=6846

4 Jee-Lyn García J, Sharif MZ. Black lives matter: a commentary on racism and public health. Am J Public Health 2015;105:e27-30.

5 Bailey ZD, Krieger N, Agénor M, et al. Structural racism and health inequities in the USA: evidence and interventions. Lancet 2017;389:1453-63.

6 Wildeman C, Wang EA, incarceration M. Mass incarceration, public health, and widening inequality in the USA. Lancet 2017;389:1464-74.

7 Winkelman TNA, Chang VW, Binswanger IA. Health, Polysubstance use, and criminal justice involvement among adults with varying levels of opioid use. JAMA Netw Open 2018;1:e180558.

8 Nellis A. The color of justice: racial and ethnic disparity in state prisons [Internet]. The sentencing project, 2016. Available: https:// www.sentencingproject.org/publications/color-of-justice-racial-andethnic-disparity-in-state-prisons/ [Accessed 09 Oct 2020].

9 Reif S, Safley D, McAllaster C, et al. State of HIV in the US deep South. J Community Health 2017:42:844-53.

10 Alexander M. The new Jim Crow: mass incarceration in the age of colorblindness. The New Press, 2010

11 Spaulding AC, Seals RM, Page MJ, et al. Hiv/Aids among inmates of and releasees from US correctional facilities, 2006: declining share of epidemic but persistent public health opportunity. PLoS One 2009;4:e7558.

12 Maruschak L. Hiv in prisons 2001-2010, 2012.

13 Adams LM, Kendall S, Smith A, et al. Hiv risk behaviors of male and female jail inmates prior to incarceration and one year post-release. AIDS Behav 2013;17:2685-94.

14 Khan MR, Wohl DA, Weir SS, et al. Incarceration and risky sexual partnerships in a southern us City. J Urban Health 2008;85:100-13.

15 Wohl DA. Hiv and mass incarceration: where infectious diseases and social justice meet. N C Med J 2016;77:359-64.

16 Centers for Disease Control and Prevention. Hiv in the southern United States 2016;29:1-4.

17 Adimora AA, Schoenbach VJ, Doherty IA. Hiv and African Americans in the southern United States: sexual networks and social context. Sex Transm Dis 2006;33:S39-45.

18 Adimora AA, Schoenbach VJ, Doherty IA. Concurrent sexual partnerships among men in the United States. Am J Public Health 2007:97:2230-7.

19 Oser CB, Pullen E, Stevens-Watkins D, et al. African American women and sexually transmitted infections: the contextual influence of unbalanced sex ratios and individual risk behaviors. J Drug Issues 2017;47:543-61.

20 Rueda S, Mitra S, Chen S, et al. Examining the associations between HIV-related stigma and health outcomes in people living with HIV/ AIDS: a series of meta-analyses. BMJ Open 2016;6:e011453.

21 The Centers for Disease Control and Prevention. 2019 National HIV surveillance system reports [Internet], 2021. Available: https://www. cdc.gov/nchhstp/newsroom/2021/2019-national-hiv-surveillancesystem-reports.html [Accessed 2 Jun 2021].
22 Grant RM, Lama JR, Anderson PL, et al. Preexposure chemoprophylaxis for HIV prevention in men who have sex with men. N Engl J Med Overseas Ed 2010;363:2587-99.

23 Baeten JM, Donnell D, Ndase P, et al. Antiretroviral prophylaxis for HIV prevention in heterosexual men and women. N Engl J Med 2012;367:399-410.

24 Nunn AS, Brinkley-Rubinstein L, Oldenburg CE, et al. Defining the HIV pre-exposure prophylaxis care continuum. AIDS 2017;31:731-4.

25 Huang Y, Zhu Y, Smith D. HIV preexposure prophylaxis, by race and ethnicity - United States, 2014-2016, 2018.

26 The Centers for Disease Control and Prevention. HIV prevention pill not reaching most Americans who could benefit - especially people of color [Internet], 2018. Available: https://www.cdc.gov/nchhstp/ newsroom/2018/croi-2018-PrEP-press-release.html [Accessed 6 Nov 2020].

27 World Health Organization. Guideline on when to start antiretroviral therapy and on pre-exposure prophylaxis for HIV. Geneva, 2015.

28 Bronfenbrenner U. Ecological Models of Human Development. In: Gauvain M, Cole M, eds. Readings on the development of children. 4 edn. New York: Worth Publishers, 1979: 3-8.

29 National Institute of Minority Health Disparities. NIMHD minority health and health disparities research framework health disparity populations: Race/ethnicity, low Ses, rural, sexual/gender minority other fundamental characteristic: Sex/gender, disability, geographic region. Washington D.C, 2018.

30 Williams DR, Jackson JS, Yan Y. Racial differences in physical and mental health: socio-economic status, stress and discrimination. J Health Psychol 1997;2:335-51.

31 Zimet GD, Dahlem NW, Zimet SG, et al. The multidimensional scale of perceived social support. J Pers Assess 1988;52:30-41.

32 Knighton Joi-Sheree', Stevens-Watkins D, Oser C, et al. Perceived risk of HIV infection among drug-using African American male prisoners: one year after community re-entry. Subst Use Misuse 2016;51:1610-8.

33 Cepeda A, Nowotny KM, Frankeberger J, et al. Biological risk and infection profiles of young adult male Mexican American gang members. Public Health Rep 2018;133:551-8.

34 McLellan AT, Luborsky L, Woody GE. An improved diagnostic evaluation instrument for substance abuse patients: The Addiction Severity Index. Vol. 168, Journal of Nervous and Mental Disease. In: McLellan A, ed. Thomas: drug dependence treatment service, Veterans administration Hospital, University and woodland avenues. Philadelphia, PA, US: Lippincott Williams \& Wilkins, 1980: 19104. 26-33.

35 Holt M, Murphy DA, Callander D, et al. Willingness to use HIV pre-exposure prophylaxis and the likelihood of decreased condom use are both associated with unprotected anal intercourse and the perceived likelihood of becoming HIV positive among Australian gay and bisexual men. Sex Transm Infect 2012;88:258-63.

36 Singer JD. Using SAS proC mixed to fit multilevel models, hierarchical models, and individual growth models. Journal of Educational and Behavioral Statistics 1998;23:323-55.

37 Mcculloch CE, Neuhaus JM. Generalized linear mixed models [Internet]. Wiley StatsRef: Statistics Reference Online, 2014.

38 Buchanan AL, Hudgens MG, Cole SR, et al. Worth the weight: using inverse probability weighted COX models in AIDS research. AIDS Res Hum Retroviruses 2014;30:1170-7.

39 Lesko CR, Edwards JK, Cole SR, et al. When to Censor? Am J Epidemiol 2018;187:623-32.

40 Seaman SR, White IR. Review of inverse probability weighting for dealing with missing data. Stat Methods Med Res 2013;22:278-95.

41 Azur MJ, Stuart EA, Frangakis C, et al. Multiple imputation by chained equations: what is it and how does it work? Int J Methods Psychiatr Res 2011;20:40-9.

42 Huque $\mathrm{MH}$, Carlin JB, Simpson JA, et al. A comparison of multiple imputation methods for missing data in longitudinal studies. BMC Med Res Methodol 2018;18:168.

43 Thomas DR. A general inductive approach for analyzing qualitative evaluation data. Am J Eval 2006;27:237-46.

44 Montgomery MC, Oldenburg CE, Nunn AS, et al. Adherence to preexposure prophylaxis for HIV prevention in a clinical setting. PLOS One 2016;11:e0157742. 